\title{
Evaluated the prognosis of patients with COVID-19
}

\author{
Meng $\mathrm{Wu}^{1}$, neng $\mathrm{Wei}^{1}$, lin $\mathrm{Mi}^{1}$, liang Zhou ${ }^{1}$, Ying Wang ${ }^{1}$, and Bin Ren ${ }^{1}$ \\ ${ }^{1}$ Wuhan University Zhongnan Hospital
}

August 3, 2020

\begin{abstract}
Background: Echocardiography derived myocardial performance may be impaired.Objectives: To evaluate cardiac involvement including cardiac biomarkers, echocardiographic findings in patients with COVID-19 and to explore the effect of cardiac impairment on short-term outcome.Methods: This study cohort was conducted from February 9, 2020, to March 28, 2020, in a single center at Wuhan Leishenshan Hospital. 213 patients whose echocardiography were included. Demographic data, laboratory results, echocardiographic findings were analyzed. Results:Among 213 patients confirmed with COVID-19,150 noncritical patients and 35 critical patients were discharged. 28 critical patients needed invasive mechanical ventilation, 16 of whom died during the hospitalization, and another 12 patients were transferred for continued treatment with tracheotomy. The incidence of cardiovascular complications including acute myocardial injury, arrhythmia and acute myocardial infarction was higher in critical group. A total of 108 patients had abnormities on echocardiography. 26(12.2\%) patients have presented the signs of pulmonary hypertension and the presence of pulmonary hypertension in critical group was higher than that in non-critical group The cardiac biomarkers at admission in critical patients were significantly higher compared with non-critical patients.Multivariate analysis showed high-sensitivity cardiac troponin I elevation and echocardiographic signs of pulmonary hypertension ere independent risk factors of adverse outcome.CONCLUSIONS: The elevation of cardiac markers and echocardiographic signs of pulmonary hypertension are risk factors of adverse outcome in patients with COVID-19. It's meaningful to combine echocardiography with cardiac markers to evaluate the prognosis of patients with COVID-19.
\end{abstract}

\section{Background}

A new coronavirus disease 2019(2019-nCoV)first occurred in Wuhan, Hubei Province in December 2019 and then broken out rapidly. Before long, the new disease had gained wide attention all over the world. By now, cardiac damage has been found in many patients with COVID-19 according to the cardiac correlated laboratory examination abnormalities and the electrocardiogram findings ${ }^{[1]}$. Nevertheless, few studies had reported the echocardiographic signs about cardiac injury. Therefore, it is meaningful to comprehensively analyze the changes from cardiac biomarkers and echocardiographic findings.

\section{Methods}

\section{Study design and participants}

This is a retrospective cohort study of patients who were confirmed with COVID-19 admitted to Wuhan Leishenshan Hospital which was supervised by the Zhongnan Hospital of Wuhan University in China from February 9, 2020 to March 28, 2020. A total of 213 patients whose echocardiography were performed within 72 hours after admission were included. The final date of follow-up was April 15,2020.

All patients included in the study were diagnosed by the respiratory pathogen nucleic acid test - chest computed tomographic (CT) scans and typical clinical presentation. We divided the patients into two groups according to severity of illness: non-critical group and critical group. The critical patients must meet the following criteria: respiratory failure, shock, multiple organ failure, requiring mechanical ventilation or intensive care management. 
This study was approved by the institutional ethics board of Zhongnan Hospital of Wuhan University (No. 2020042). The ethics commission approved the application for exemption from the requirement for informed consent.

\section{Data Collection}

The clinical data of these patients including laboratory results, clinical outcomes were collected and analyzed from electronic medical records. The echocardiographic image acquisition, measurement and results were acquired from the imaging management system in ultrasound department. Bedside echocardiographic examinations were performed in critical patients with Mindray portable machine. Siemens Machine (ACUSON OXANA1) was used for image acquisition of non-critical patients. The cardiac biomarkers were traced and assessed on days $1,3,7,14,21$ and 30 .

\section{Definitions}

Cardiac injury was defined if the serum level of high-sensitivity troponin I (hs-cTnI) were above the 99th percentile upper reference limit ${ }^{[2]}$. The illness severity of COVID-19 was defined according to the Chinese management guideline for COVID-19 (version 7.0). Abnormalities on echocardiography defined as cardiac chambers enlargement, cardiac dysfunction (defined as left ventricular ejection fraction $<50 \%$ ), segment wall motion abnormality, left ventricular wall thickening $(>10 \mathrm{~mm})$ and/or pericardial effusion. Right ventricular systolic function was represented by the tricuspid anterior plane systolic excursion (TAPSE). Echocardiographic signs of pulmonary hypertension includes the following:the peak tricuspid regurgitation velocity is over $2.8 \mathrm{~m} / \mathrm{s}$ accompanied by the basal diameter ratio of right ventricle/left ventricle $>1.0$; the acceleration time of pulmonary artery is over $105 \mathrm{msec}$ and/or mild systolic notching; the inferior cava diameter is more than $21 \mathrm{~mm}$ with decreased inspiratory collapse $(<50 \%$ in the condition of sniffing or $<20 \%$ in quiet inspiration $)^{[3]}$.

\section{Statistical analysis}

The statistical analysis was done using SPSS (version 26). Continuous and categorical variables are presented as the median (IQR) and $\mathrm{n}(\%)$, respectively. The Mann-Whitney $\mathrm{U}$ test, $\chi^{2}$ test, or independent sample $\mathrm{t}$ test were used to compare differences between critical and non-critical patients where appropriate. We use univariable and multivariable logistic regression analysis to explore risk factors associated with adverse outcome. Considering the total number of patients with adverse outcome $(n=28)$ in our study and to ensure stability of the multivariable logistic regression model, four variables were included in the analysis. Our model included age, comorbidity of cardiac disease, hs-cTnI elevation and echocardiographic signs of pulmonary hypertension based on previous studies and results of baseline characteristics comparison. For all analysis, statistical significance was considered $p<0.05$.

\section{Results}

\section{Baseline clinical characteristics}

213 patients diagnosed with COVID-19 were included in the final analysis,including 150 non-critical patients and 63 critical patients. Of 213 patients, $110(51.6 \%)$ were male. The median (IQR) age was 63.0(56.073.0). The median age of critical group was significantly higher than that of non-critical group (77.0 vs 63.0 years, respectively; $p$ ¡0.001). There was significant difference in sex between two groups $(p ; 0.01)$ and most of critical patients were male. Hypertension (52.6\%), cardiac disease (21.6\%), diabetes (19.2\%) were the three most common comorbidities. The presence of cardiac disease, cerebrovascular disease and chronic kidney disease in critical group was all higher than that in non-critical group $(p ; 0.05)$. The incidence of cardiovascular complications including acute myocardial injury, arrhythmia and acute myocardial infarction was higher in critical group than non-critical group. 5 of 63 critical patients developed acute myocardial infarction. (Table 1)

\section{Echocardiographic Characteristics}


A total of 108 patients $(50.7 \%)$ had abnormities on echocardiography. The incidence of cardiac chamber enlargement, wall thickening, cardiac disfunction and pericardial effusion in critical patients was significantly higher than that in non-critical patients $(p ; 0.05)$. There were $22(10.3 \%)$ patients with LVEF $<50 \%$. The critical patients demonstrated lower median LVEF than non-critical patients $(p ; 0.01)$. There was no significant difference of the maximum depth of pericardial effusion and tricuspid annular plane systolic excursion(TAPSE) $(p$ i.0.05)between two groups. 26(12.2\%) patients have presented the signs of pulmonary hypertension and the presence of pulmonary hypertension in critical group was higher than that in noncritical group $(p ; 0.001)$.(Table 2,Figure 1-3)

\section{Laboratory Findings on Admission}

Compared with non-critical patients, the inflammatory biomarkers in critical patients including c-reactive protein (CRP), procalcitonin (PCT), interleukin-2 receptor (IL-2R), $\alpha$-tumor necrosis factor (TNF-A) and Interleukin-6 (IL-6) were significantly higher $(p ; 0.05)$. The cardiac biomarkers in critical patients including creatine kinase MB (CK-MB), high-sensitivity troponin I (hs-cTnI), creatine kinase (CK), lactate dehydrogenase $(\mathrm{LDH}), \alpha$ - hydroxybutyrate dehydrogenase $(\mathrm{HBDH})$ and brain natriuretic peptide (BNP) were significantly higher than non-critical patients $(p ; 0.001)$. There were numerous differences in other baseline laboratory findings between two groups, including longer prothrombin time, higher levels of D-dimer and aspartate aminotransferase (AST) (Table 3).

\section{Dynamic changes of cardiac biomarkers levels during hospitalization}

All patients were divided into three groups (non-critical group, critical-survivor group and criticalnonsurvivor group to record and compare dynamic changes of cardiac biomarkers. BNP increased rapidly after the 14th day in critical-nonsurvivor group but decreased respectively from the 3th day and 7th day in the non-critical and critical-survivor groups. Levels of hs-cTnI was clearly elevated from the 14th day and rapidly decreased from the 21th day in critical-survivor group and critical-nonsurvivor group, but for non-critical group, it gradually decreased after the peak on the 3th day. Differences among three groups were significant for LDH, CK-MB, HBDH throughout the clinical course. LDH and HBDH increased for all patients in the early stage of illness, then decreased for non-critical and critical survivors but rapidly increased for nonsurvivors in the late stage of illness.

\section{Risk factors of adverse outcome}

At the end of follow-up, all non-critical patients and 35 of 63 critical patients were discharged. 28 critical patients needed invasive mechanical ventilation, 16 of whom died during the hospitalization, and another 12 patients were transferred to Zhongnan Hospital of Wuhan University with tracheotomy. By multivariate logistic regression, we found that hs-cTnI elevation (OR 6.069, 95\% CI 2.358-15.615; $p=0.000$ ) and echocardiographic signs of pulmonary hypertension of pulmonary hypertension (OR 3.635, 95\% CI 1.257-10.509; $p$ $=0.017$ ) may be the independent risk factors of adverse outcome. (Table 4)

\section{CONCLUSIONS:}

The changes of cardiac structure and function as well as abnormalities of electrocardiogram in critical patients were relatively more obvious. The older age- elevation of cardiac markers and echocardiographic signs of pulmonary hypertension may be the risk factors of adverse outcome in patients with COVID-19. It's meaningful to combine echocardiography with cardiac markers to evaluate the prognosis of patients with COVID-19.

\section{Discussion:}

This article aims to evaluate cardiac involvement including cardiac biomarkers, echocardiographic findings as well as cardiovascular complications in patients with COVID-19, and to explore the association of cardiac impairment and short-term outcome.

Advanced age has been reported as a predictor of mortality in SARS, MERS and COVID- 19 $9^{[2,4,5]}$. There is no doubt that older age was a correlation of adverse outcome in this $\operatorname{study}(P ; 0.05)$. The ef- 
fect of myocardial injury on adverse outcomes and/or in-hospital mortality has been reported in the recent literature $^{[1]}$. In the current study, $33.5 \%$ patients exhibited myocardial injury as demonstrated by elevation of hs-TnI levels, and the incidence of myocardial injury in critical patients was significantly higher than that in non-critical patients $(74.2 \%$ vs $12.0 \%)$. Multivariate analysis showed that increased baseline hs-TnI level was one of the independent risk factors for adverse outcomes. The rapid increasing level of BNP, LDH and HBDH in the late stage of illness in fatal cases suggested that myocardial injury played a greater role in the fatal outcome of COVID-19. The recent study found that the in-hospital mortality was higher in patients with elevated TNT level than that in patients with normal TNT level $(59.6 \% \text { vs } 8.9 \%)^{[6]}$. It has been reported that cardiovascular system was affected by multiple possible mechanisms. On the one hand, direct SARS-CoV-2 infection of the myocytes causing active myocarditis might also contribute to the impairment in myocardial function. A case report in which a patient infected with SARS-CoV-2, without a history of cardiovascular disease, experienced acute myocarditis manifested as increased wall thickness, diffuse biventricular hypokinesis and severe left ventricular dysfunction, with the cardiac MRI findings showing marked biventricular myocardial interstitial edema and the slow gadolinium washout ${ }^{[7]}$. On the other hand, balance between coronary perfusion and cardiac metabolic was disturbed by cytokine storm. The release of inflammatory cytokines may lead to the increased metabolic needs of peripheral tissues and organs including cardiac tissue, accompanied by coronary artery spasm or coronary microvascular dysfunction with decreased coronary blood flow, decreased oxygen supply, destabilization of coronary plaque, and microthrombogenesis ${ }^{[8]}$. In our study cohort, critical patients demonstrated higher level of Inflammatory biomarkers including C-reactive protein , procalcitonin , Interleukin-2 receptor, $\alpha$-tumor necrosis factor , and interleukin- 6 than non-critical patients. To a certain degree, it partly explained probable cause for five patients in this cohort complicating by acute myocardial infarction after infected with SARS-CoV-2. The atherosclerotic plaques of coronary arteries are prone to rupture because their inflammatory activity is intensified in the systemic inflammatory response ${ }^{[9]}$.

There are few literatures referred to the echocardiographic signs in patients with COVID-19. The enlargement of right atrium, right ventricle and pulmonary hypertension may be related to the severity of pulmonary lesions and acute respiratory distress syndrome (ARDS). ARDS and severe lung injury impact cardiac hemodynamics through pulmonary vascular dysfunction, which frequently manifest pulmonary hypertension and RV overload caused by inflammatory factors, hypoxia-induced mediators, hypercapnia-induced vasoconstriction, thrombosis, and pulmonary vascular remodeling ${ }^{[10]}$. However, the effect of pulmonary hypertension on the prognosis of patients with ARDS is controversial. Squara et al. ${ }^{[11]}$ reported that fatal cases had a higher mean pulmonary artery pressure in a study of 586 patients with ARDS. On the contrary, Osman et al. ${ }^{[12]}$ found that right heart failure / pulmonary hypertension didn't have effect on adverse outcome in patients with ARDS. However, in our cohort, increased pulmonary artery systolic pressure was also one of the independent risk factors for adverse outcomes. In addition to being associated with the cytokine storm damage to the lung, the myocardial ischemia leading to the hypoperfusion of the right ventricle may be also the cause of pulmonary hypertension. The COVID-19 has been reported resembled the severe acute respiratory syndrome coronavirus (SARS-CoV) which caused an outbreak in 2003. Siu-fung Yiu et al. observed echocardiographic reversible, subclinical diastolic left ventricular impairment without systolic involvement in a cohort of acute SARS infection irrespective of the severity of their disease ${ }^{[13]}$. A lower mean left ventricular ejection fraction (LVEF) was found in patients who required mechanical ventilation. In line with their findings, the critical patients in our cohort demonstrated lower median LVEF than non-critical patients. However, no significant pulmonary hypertension was found in their study. Researchers speculated that the temporary changes in the echocardiographic parameters in the acute phase might be caused by the cytokine storm resulting from an excessive host immune response to SARS virus infection ${ }^{[14]}$. However, the changes in the echocardiographic parameters were generally nonspecific, a more severe impairment of left ventricular function in patients with COVID-19, like the 22 patients with cardiac disfunction with LVEF ¡50\%, may suggest an exacerbation of the underlying heart disease or the presence of other secondary causes of left ventricular impairment. Segmental wall motion abnormalities occurred in patients with history of coronary heart disease and/or complications of myocardial infarction in the course of disease. Pericardial effusion was more likely to be related to systemic inflammatory reaction and wall thickening was more likely to be related to pre-existing cardiovascular disease such as hypertension or other metabolic diseases impaired the 
myocardium. Nevertheless, echocardiographic signs didn't exhibit the typical myocarditis change.

\section{Limitation}

Our study has several limitations. First, this is a single-center retrospective study. Our 213 patients may not represent the whole population, the study sample size was limited. Second,only one echocardiographic finding cannot represent the dynamic changes of the heart,if possible,dynamic monitoring is helpful.Third,we did not focus on the early changes of heart using new technology such as Speckle tracking.

\section{Reference}

[1] Shi S, Qin M, Shen B, et al. Association of Cardiac Injury With Mortality in Hospitalized Patients With COVID-19 in Wuhan, China[J]. JAMA Cardiol, 2020.

[2] Zhou F, Yu T, Du R, et al. Clinical course and risk factors for mortality of adult inpatients with COVID-19 in Wuhan, China: a retrospective cohort study[J]. The Lancet, 2020,395(10229):1054-1062.

[3] Galie N, Humbert M, Vachiery J L, et al. [2015 ESC/ERS Guidelines for the diagnosis and treatment of pulmonary hypertension][J]. Kardiol Pol, 2015,73(12):1127-1206.

[4] Peiris J, Chu C M, Cheng V, et al. Clinical progression and viral load in a community outbreak of coronavirus-associated SARS pneumonia: a prospective study[J]. The Lancet, 2003,361(9371):1767-1772.

[5] Hong K H, Choi J P, Hong S H, et al. Predictors of mortality in Middle East respiratory syndrome (MERS)[J]. Thorax, 2018,73(3):286-289.

[6] Guo T, Fan Y, Chen M, et al. Cardiovascular Implications of Fatal Outcomes of Patients With Coronavirus Disease 2019 (COVID-19) [J]. JAMA Cardiol, 2020, [Epub ahead of print].

[7] Inciardi R M, Lupi L, Zaccone G, et al. Cardiac Involvement in a Patient With Coronavirus Disease 2019 (COVID-19)[J]. JAMA Cardiol, 2020.

[8] Huang C, Wang Y, Li X, et al. Clinical features of patients infected with 2019 novel coronavirus in Wuhan, China[J]. The Lancet, 2020,395(10223):497-506.

[9] Madjid M, Vela D, Khalili-Tabrizi H, et al. Systemic infections cause exaggerated local inflammation in atherosclerotic coronary arteries: clues to the triggering effect of acute infections on acute coronary syndromes[J]. Tex Heart Inst J, 2007,34(1):11-18.

[10] Namendys-Silva S, Santos-Martínez L, Pulido T, et al. Pulmonary Hypertension Associated With Acute Respiratory Distress Syndrome[J]. Chest, 2013,144(4, Supplement):376A.

[11] Squara P, Dhainaut J F, Artigas A, et al. Hemodynamic profile in severe ARDS: results of the European Collaborative ARDS Study[J]. Intensive Care Med, 1998,24(10):1018-1028.

[12] Osman D, Monnet X, Castelain V, et al. Incidence and prognostic value of right ventricular failure in acute respiratory distress syndrome[J]. Intensive Care Med, 2009,35(1):69-76.

[13] Li S S, Cheng C W, Fu C L, et al. Left ventricular performance in patients with severe acute respiratory syndrome: a 30-day echocardiographic follow-up study[J]. Circulation, 2003,108(15):1798-1803.

[14] Peiris JS, Chu CM, Cheng VC, et al. Clinical progression and viral load in a community outbreak of coronavirus-associated SARS pneumonia: a prospective study[J].Lancet, 2003,361(9371):1767-72.

Figure legend:

Figure1 .A female patient, 77years old, was admitted to WuHan Lei Shen Shan hospital with fatigue and right abdominal pain for 30 days.Echocardiography showed that the left atrium was enlarged, the movement of the ventricular wall decreased, and the left ventricular systolic function decreased. 
Figure2、3 . A male patient, 81 years old, was admitted to WuHan Lei Shen Shan hospital with cough and chest stuffiness for 2 weeks.Echocardiography showed pericardial effusion $(9 \mathrm{~mm}$ behind the left posterior wall of the left ventricle), mild tricuspid regurgitation, maximum reflux pressure about $36 \mathrm{mmHg}$, and pulmonaryarterialsystolicpressure was estimated to be about $46 \mathrm{mmHg}$, therefore this patient has mild pulmonary hypertension.

Figure 4 Dynamic changes of cardiac biomarkers during hospitalization. Figure shows dynamic changes in high-sensitivity cardiac troponin I $(\mathrm{ng} / \mathrm{ml})(\mathrm{A})$, Brain natriuretic peptide $(\mathrm{pg} / \mathrm{ml})(\mathrm{B})$, Creatine kinase MB (C), Lactate dehydrogenase (U/L) (D), $\alpha$ - hydroxybutyrate dehydrogenase(E).

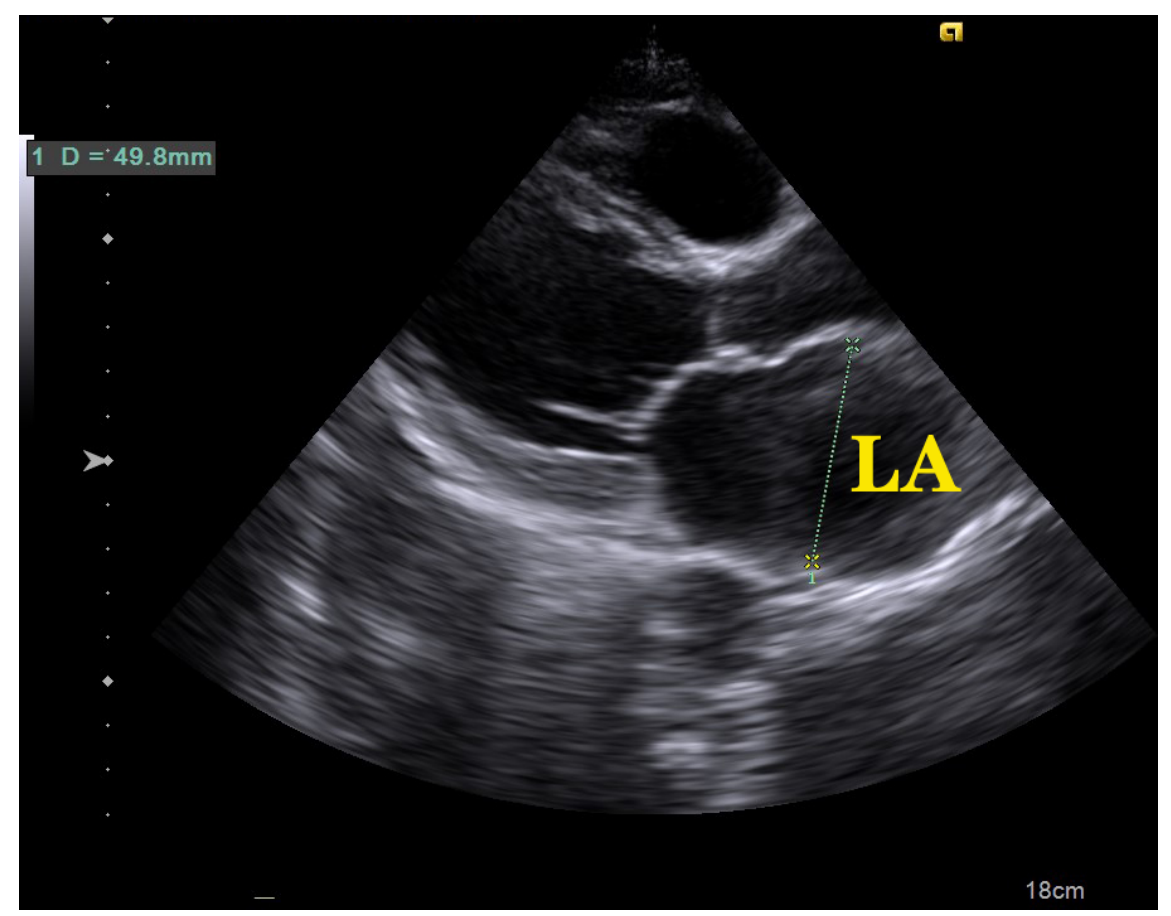



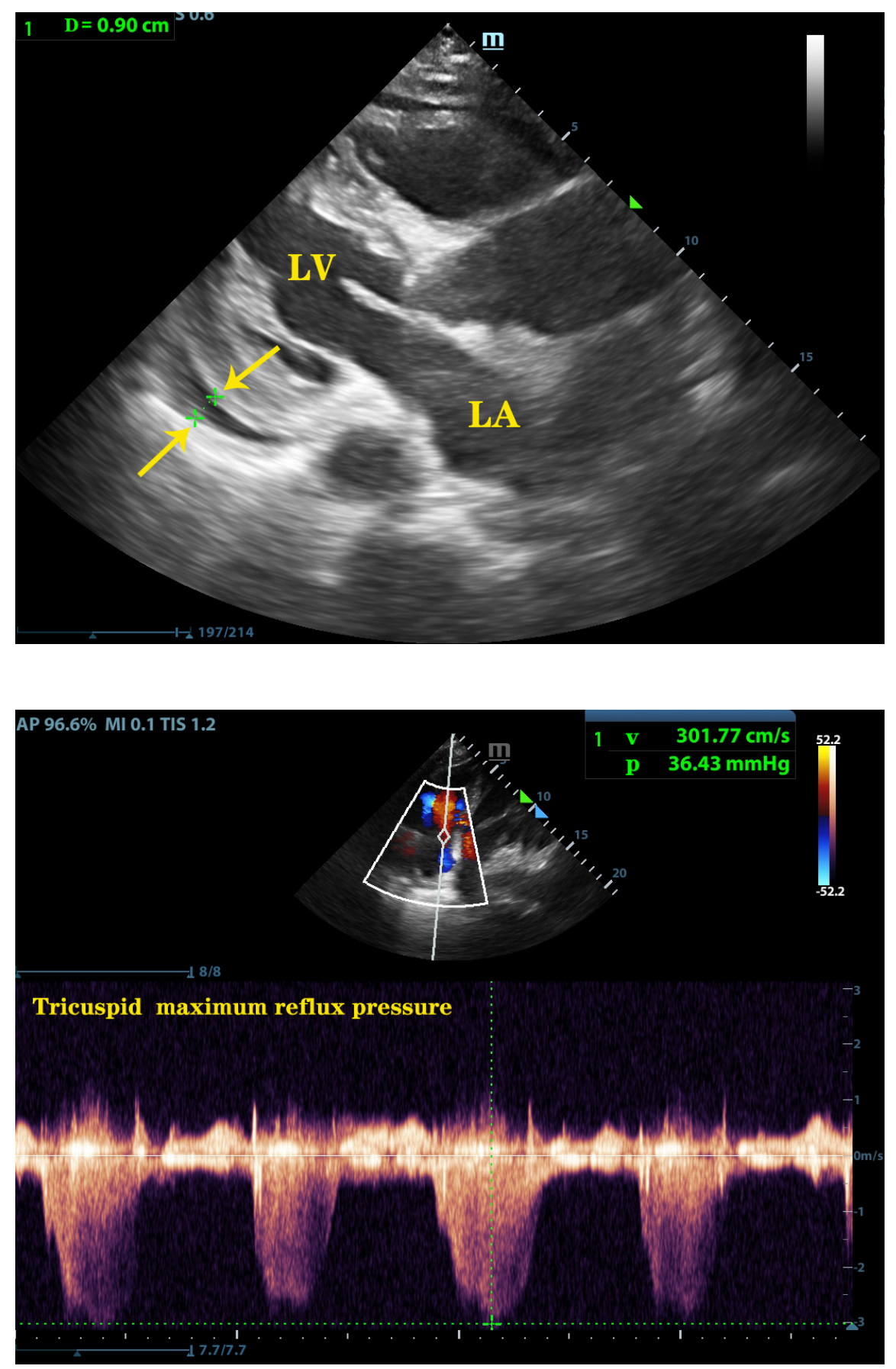

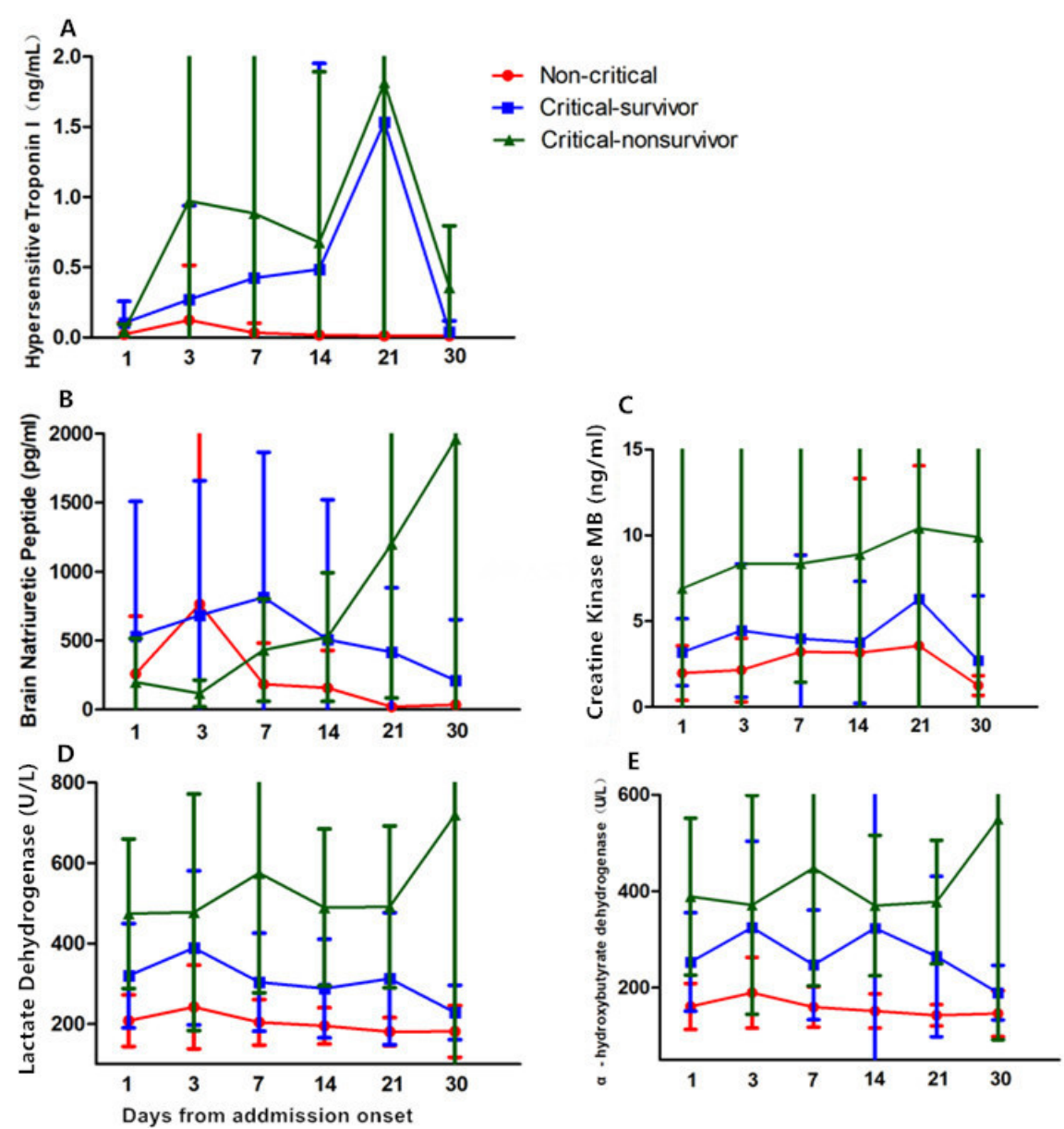